\title{
Light in Medicine: The Interplay of Chemistry and Light
}

\author{
Claire S. Allardyce*, Roland Bays, and Nicolas Thévenaz
}

\begin{abstract}
Photodynamic therapy (PDT) has had mixed reception in the clinic, with most success stories being based on the ablative capacity of PDT. In these applications, maximal combinations of light and an exogenous photosensitiser are used to generate high levels of reactive oxygen species (ROS) that induce cell death either directly via necrosis or indirectly via vascular damage. However, recent advances in understanding the complex role of ROS in cell signalling have revealed potential new applications for PDT. For example, the proliferative effects of low level ROS could be applied to wound healing or immunomodulation. These effects should also be considered in the ablative applications. With the decades of chemical advances for ablative PDT at hand including targeting mechanisms to diseased cells and subcellular locations, optimisation of light absorption, and carrier mechanisms that modulate the therapeutic response - the application of PDT to other types of treatment could be relatively rapid. This review serves to summarise some of these developments and suggest future directions.
\end{abstract}

Keywords: Apoptosis · Autophagy · Photodynamic therapy $\cdot$ Reactive oxygen species $\cdot$ Wound healing

\section{Introduction}

Photodynamic therapy (PDT) involves activating a photosensitiser (PS) with light. The activated PS interacts with molecular oxygen to produce radicals and activated oxygen species, collectively termed reactive oxygen species (ROS). The consensus during development of the technique in the 1980s and 1990s was to optimise ROS production, particularly singlet oxygen species, to trigger apoptosis in diseased cells. Knowing that necrosis was triggered at slightly higher doses allowed the design of strategies that employed the maximal light and drug doses to produce high ROS levels, with a rapidly photobleaching PS to regulate the effect such that apoptosis was favoured. However, improved understanding of the mechanism of ROS signalling has led to a new approach that considers cell death via necrosis and immunologically induced cell death carrying some advantages, particularly when there may be diseased cells outside the treatment zone. Furthermore, PS that do not produce singlet oxygen have successfully completed phase III clinical trials. The improved understanding of signalling has also opened

${ }^{*}$ Correspondence: Dr. C. S. Allardyce the development of PDT to new therapies, including those where cell proliferation and for the future recolonisation of diseased tissues via the release of endothelial progenitor cells from the bone marrow can be beneficial.

The PS-induced ROS response is both quantitative and qualitative. The amount of ROS produced triggers different responses ranging from necrosis through apoptosis, macroautophagy, and chaperone-mediated autophagy to cell signalling events. Some responses lead to cell death, others are cytostatic, but the responses to lower levels of ROS are often proliferative and this type of signalling is known to be involved in angiogenesis and metastasis. On a qualitative level, ROS includes a combination of species: including singlet oxygen, hydroxyl radicals, and superoxide ions. Some PS favour the production of one of these species over another and each has a subtly different effect on the surrounding tissues. Although it is widely believed singlet oxygen - usually the first ROS formed on PS excitation - is the marker of PDT, ${ }^{[1]}$ in aqueous solution the bacteriopheophorbide WST11 (soluble TOOKAD; Fig. 1), which

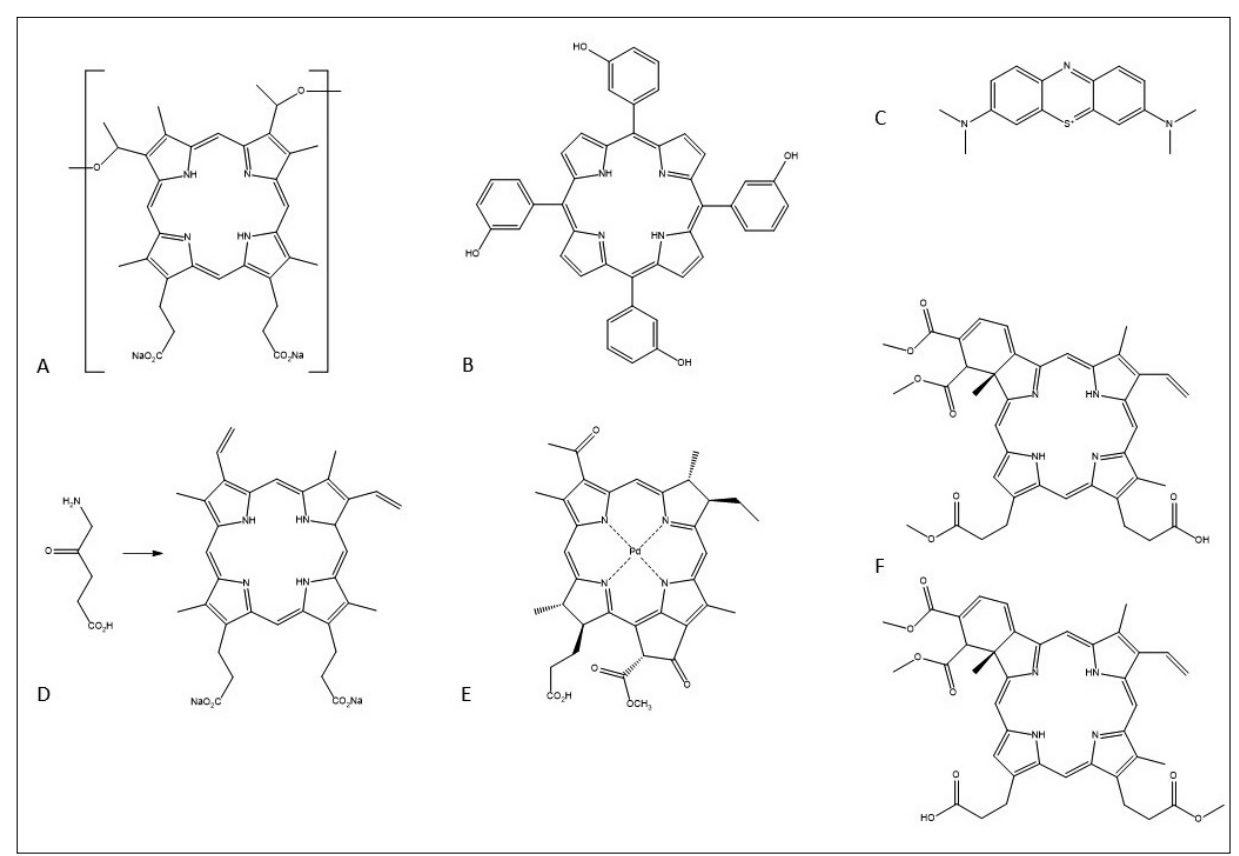

Fig.1. Chemical Structures of some of the most well-known photosensitisers. A. Photofrin ${ }^{\oplus}$; B. Foscan ${ }^{\circledR}$; C. methylene blue; D. ALA and Protoporphyrin IX; E. soluble TOOKAD ${ }^{\circledR}$; F. Visudyne ${ }^{\circledR}$. 
has recently completed phase III clinical trials for prostate cancer, only generates superoxide and hydroxyl radicals. ${ }^{[1,2]}$ It is now accepted that singlet oxygen is critical for PDT and superoxide anion radicals, hydrogen peroxide, and hydroxyl radicals are important contributors to efficacy. ${ }^{[3]}$

\section{From Bench to Bedside and Back Again}

The first PDT effects reported were cytostatic and ablative. Back in the early 1900s PDT was pioneered as a treatment modality for microbial infection and, shortly after, cancer. The results were based on the clinical outcome of treatment and although it was understood that the mechanism involves a PS, light and oxygen, little else was known.

The technique was not extensively investigated until the late 1970s when technology was improving and characterisation of molecular pathways was becoming possible. The first generation PS was hemoporfrin, commercialised under the name Photofrin ${ }^{\circledR}$ (Fig. 1) and the first formulation contained over 60 different species. In the years that followed the PS was refined and treatments approved in some countries for several indications including the palliation of obstructing esophageal cancer, microinvasive endobronchial nonsmall-cell lung cancer and the ablation of high-grade dysplasia in Barrett's esophagus. Still, therapy was not without its problems, most notably the long duration of photosensitivity after the treatment and the fact that the wavelength of light used to excite Photofrin ${ }^{\circledR}$ is absorbed by naturally occurring chromophores, resulting in a limited treatment depth.

Despite approval of Photofrin ${ }^{\circledR}$-PDT, the further development of the PS remained a priority research effort. Amongst the second generation PSs were chlorins and phthalocyanines offering improved properties with respect to activation as the absorption of light was at wavelengths in the biological absorption window. This window exists in the infrared region of the light spectrum, where the collective contribution of biological chromophores is lowest allowing for further propagation from the site of illumination through the tissue. In some applications, such as bacterial disinfection, surficial treatment is preferential, however with treatments for cancer under the spotlight, deeper propagation was sought to ensure all diseased tissue was ablated. Chlorins, such as Foscan ${ }^{\circledR}$ (Fig. 1), are approved for indications such as head and neck cancer in some countries. However, these species still exhibit long clearance times and therefore extended phototoxicity.

\section{More Bench Work}

The third generation PSs are those with integrated targeting mechanisms. The PS for ablative techniques must also be effective at generating ROS. ${ }^{[4-8]}$ PDT selectivity initially focused on the location of light irradiation, drug uptake and the fact that normal cells often have a higher tolerance to additional ROS and therefore survive the treatment. ${ }^{[9,10]}$ However, there are some widely acknowledged limitations, particularly general photosensitivity after treatment and the heterogeneity in the light and PS dose resulting in an uneven ROS production across the treatment site, with the latter becoming more of a concern as the full spectrum of ROS response is unveiled.

Cells exhibiting rapid metabolism and growth have an accordingly accelerated uptake of metabolic precursors, which includes some types of PSs. Therefore, these complexes have the tendency to accumulate in microbially infected and cancer cells. The environment around the tumour cell also promotes selectivity, with altered vascularisation preferentially retaining PSs within the diseased tissues and the hypoxic environment selecting for species, such as methylene blue (MB; Fig. 1), that require reduction before uptake. ${ }^{[11]}$ In the case of aminolevulinic acid (ALA; Fig. 1) and its derivatives, which are assembled into porphyrin species (PpIX; Fig. 1) in the cell to afford metabolic targeting, different species with subtly different photochemical properties are produced according to the profile of the cell. For example, synovial tissue assembles a species that is more hydrophobic than cartilage. However, although ALA uptake is selective enough to allow fluorescence of the assembled PpIX to guide surgical intervention, rapid photobleaching during PDT limits ROS generation and the degree of ablation. Furthermore, ALA enters neurons where the generation of ROS results in chronic pain during PDT. The pain can be managed, but prevention would be better.

In other areas of drug development, solubility has been found to be a key feature in delivery, ensuring optimal localisation of the drug at the treatment site and has therefore been, and continues to be, a key feature considered in the development of third generation PSs. Strategies developed for other applications can be applied to PSs to optimise distribution through the diseased tissue and designing systems that target the PS to specific cells. ${ }^{[12,13]}$ Already improvements in the uptake of topologically applied ALA have been made using emulsions, liposomes or nanoparticles, for example, as potential carrier vehicles. ${ }^{[4,6,7,14,15]}$ In other cases, where surficial treatments are required, inert carrier molecules can prevent diffusion and maintain an extracellular location for the PS. MB-colloids have been developed with such a goal. Although these systems display a decreased singlet oxygen quantum yield, ${ }^{[6]}$ the overall therapeutic outcome is improved.

In the development of the third generation PSs, macromolecular carriers, from nanoparticles to fullerenes, have been investigated as a means to selectively deliver the PS to the diseased tissue. Micelle-encapsulated PSs seem to prevent cell uptake, affording a vascular mode of action, ${ }^{[16]}$ whereas liposomes can deliver encapsulated drugs into the cell. Photoactivated release mechanisms have been reported where light can be used to trigger controlled spatial and temporal release. ${ }^{[17]}$ Another selective release strategy uses localised enzymes to mediate unloading. Mesoporous silica nanoparticles sealed with oligosaccharides to afford site-specific rhodamine B release have been shown to remain intact until bacterial secreted $\beta$-mannanase degrades the sealant to release the PS. [18]

Although there is much controversy about the use of nanoparticles in medicine due to possible associated health problems and challenging optimisation of biophysical properties, they have been investigated for targeted PS delivery. For example, polymeric nanoparticles have been used to encapsulate curcumin with an associated increase in fluorescence intensity and lifetime. ${ }^{[19]}$ In addition to offering tumour selection via the enhanced permeability effect and retention effect, molecular tags, such as folic acid, targeting peptides or antibodies, can be attached to the NPs, offering tissue-, cell-, or subcellular-specific delivery. ${ }^{[20]}$ The use of such carriers also opens the door to the fourth generation PSs, where effect-modulatory technologies, such as up-conversion and spatial confinement of energy, are being used to fine-tune PDT.

As with the colloidal systems, size optimisation of the carrier can be designed to prevent cellular uptake. As an example, micrometre-sized porous honeycomb-patterned thin films formed by the electrostatic interaction of Mn(III) meso-tetra(4-sulfonatophenyl) porphine chloride and dimethyldioctadecylammonium bromide exhibit more efficient antibacterial activity compared to equivalent nanoparticles. ${ }^{[21]}$ Whilst this film is more targeted toward the development of in vitro photoactivated disinfection systems, certain PDT applications may benefit from fabric-style PS systems that allow contact treatment.

Various protein constructs have been investigated as delivery vehicles for PS. Where the intact haem in myoglobin was substituted by a Zn-protoporphyrin IX ${ }^{[22]}$ or hypericin ${ }^{[23]}$ fluorescence and singlet 
oxygen photosensitising properties were preserved. The protein scaffold appears to protect the PS such that dark toxicity is reduced.

Immuno-targeting of PSs has also been explored. ${ }^{[24]}$ Monoclonal antibodies raised against tumour surface molecules were covalently tagged to PSs. Although there were early complications of confirming covalent tagging rather than absorption, the studies demonstrated selective accumulation of the PS at the target site and thereby an improved treatment outcome. For example a porphycene-anti-ICAM-1 conjugate shows specificity for diseased cells, inducing phototoxicity in inflamed, but not in healthy, microvascular endothelial cells. ${ }^{[25]}$

\section{The Fourth Generation}

With the fourth generation of PSs come the possibility of tailoring light absorption and the ROS yield for particular applications. Constructs, such as fluorophore-spacer-receptor switching systems have been engineered to enable $\mathrm{pH}$ induced activation exploiting the hypoxic environment of the tumour cells to localise the PDT effect rather than the drug itself. [26]

'Click' chemistry has been used to produce selective sensitisers, [27] including a selectively activated photochromefluorophore dyad combining a fluorescent dye and a photochromic diarylethene. The open form exhibits photochroism and fluorescence, whereas the closed form does not. The dyad form may be switched using alternate UV/visible irradiation. ${ }^{[28]}$ Such strategies have also been used to develop probes for singlet oxygen species. For example, in dyads composed of a singlet oxygen trap coupled to a naphthoxazole fluorescence is quenched in the native state. However, singlet oxygen leads to an oxidation reaction that liberates a fluorescent complex. ${ }^{[29]}$

PDT also offers the possibility of combining diagnosis and therapy within a single species. Real-time feedback on the pharmacokinetics and specific localisation based on the intrinsic fluorescence of some, but not all, PSs allows personalised medicine that overcomes some of the PDT variability due to the different drug uptake and light absorption and propagation properties. [30]

The current focus in the development of fourth generation PSs includes integrated systems offering the clinical imaging of tumour tissue, targeted delivery and the potential of multimodal biomedical theranostic nanoplatforms. ${ }^{[31]}$ For clinical applications though, simple and stable formulations are preferred. In the case of PDT there are several variables: light, oxygen and PS concentration. One of these factors is limiting. When light determines the maximum effect, treatment is limited to the propagation of light through tissue (see below). However, excess light can lead to heating and changes in the collagen structure of the tissue leading to instabilities. Oxygen can be made limiting by vascular modes of activity that systematically shut down the vascular system. On the other hand, rapid exhaustion of oxygen can lead to an incomplete treatment, such that slower therapies maintaining vascularisation can be preferential. Alternatively, the PS can limit the therapeutic effect. In the case of ALA the PDT activity is capped by PS photobleaching, allowing dermatological applications where accuracy in light is not required, allowing low cost lamps to be used. Similar systems could be envisaged for treating other indications, but where the PS is not self-limiting, inaccurate light dosimetry can produce inconsistent results and risk of harm or injury to the patient.

\section{Metal Chelation}

Many new developments of PSs are based on metal chelated species. These species have altered spectral and biological properties compared to the naked equivalents and, importantly, particularly in the case of porphryins and chlorins, that have a higher probability of homogeneity at the treatment site. Intuitively, the naked porphyrin will collate metal ions in biological tissues. Intravenously administered PSs, with the exception of precursor complexes such as ALA, are likely to react in the blood. Although iron is tightly sequestered by proteins, due to its toxicity, a range of metal ions exist in biological systems with different effects on the photodynamic acstatus is a major player in controlling the PDT response. In general, chelation of parativity of PSs ${ }^{[32-34]}$ and therefore nutritional

magnetic metal ions into the PS shortens the triplet lifetimes and introduces variations in the triplet quantum yield [14,33-35] to such an extent that the photochemical reactions are prevented. ${ }^{[14]}$ Iron, copper and nickel species ${ }^{[33,35]}$ are generally reported to block photodynamic activity, whereas some other metals favourably alter the absorption properties or the quantum yield. There are exceptions to the generalisation, including copper octaethylbenzochlorin. ${ }^{[36]}$ Other metals are reported to improve the photoactivity by enhancing intersystem crossing, ${ }^{114]}$ including diamagnetic transition metals and lanthanide ions. As a result, a number of metal chelated PS systems are showing positive results, particularly zinc(II), ${ }^{[37]}$ aluminium(III), ${ }^{[38]}$ and tin(IV) ${ }^{[5,15,39]}$ complexes and a Pd(II) compound has completed phase III clinical trials. $^{[40]}$

\section{The ROS Response}

Most current treatment modalities for cancer - chemotherapeutic and radiotherapeutic agents - mediate their effect via ROS stress. Similarly, PDT mediates its anticancer effect by generating endogenous ROS, ${ }^{99}$ but unlike the other treatments, PDT is non-mutagenic so can be used repeatedly and in conjunction with chemotherapy and radiotherapy without the concern of increasing genotoxicity.

Both the duration and extent of the ROS signal is required for the therapeutic effect with sustained high ROS leading to biomolecular damage, activation of cell-cycle inhibitors ${ }^{[6,41]}$ and induction of cell death (Fig. 2). Resistance to these treatments often centres on the increased production of antioxidants, including glutathione, and deactivating enzymes, such as super oxide dismutase, and the up regulation of pumps, such as the ABC pump, to reduce the ROS levels and damage pushing the effect back towards homeostasis.

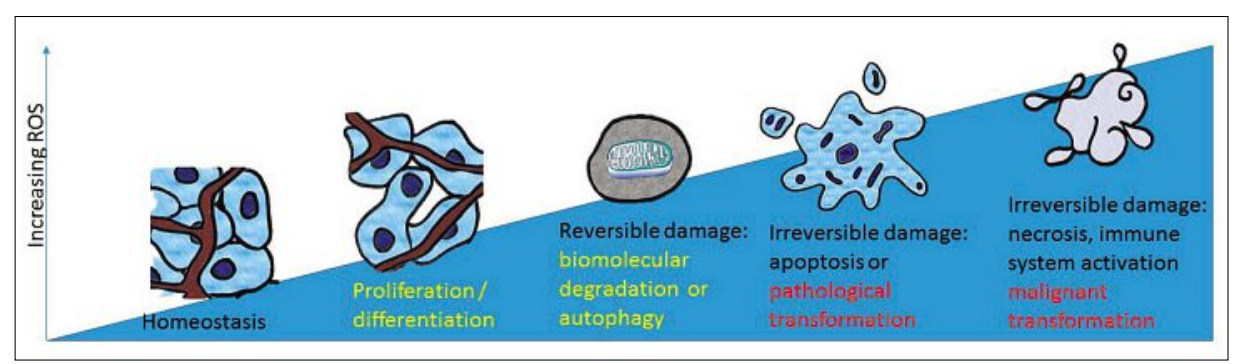

Fig. 2. Different levels of ROS elicit different cellular responses. Low levels of ROS are used in homeostasis. When levels increase beyond a threshold or the time, duration or location of ROS production exceeds the homeostasis threshold, different responses are triggered. The first response is proliferation and differentiation. Whilst these processes are involved in homeostasis, they can also be involved in disease progression. Subsequently, adaptive responses are initiated including micro and macro autophagy. When the level of ROS triggers irreversible damage, the cell responds through apoptosis or transformation. Finally, high levels of ROS, particularly that lead to DNA damage, lead to malignant transformation, where the cells survive, or necrosis. 
Glutathione also contributes to the regulation of the redox potential of the cell. Healthy cells balance the two phases of respiration - glycolysis and oxidative phosphorylation (OXPHOS) - such that there is a characteristic metabolic state. Cancer cells have a metabolic shift, known as the Warburg effect, where glycolysis occurs more rapidly than OXPHOS leading to a build-up of NADH and reduced ROS production via the mitochondrial respiratory chain. In turn, this change leads to signalling events that encompass all stages of tumour formation, i.e. initiation, promotion and progression.

ROS can also trigger autophagy (Fig. 2), specifically chaperone-mediated autophagy where ROS-damaged proteins are shunted to the lysosome and macroautophagy characterised by the formation of a double membrane autophagosome that surrounds damaged protein complexes or organelles. Although autophagy can sometimes lead to cell death, ${ }^{[2,42]}$ blocking this response has been demonstrated to enhance PDT. Chaperone-mediated autophagy is known as a survival response that promotes oncogenesis. ${ }^{[43]}$ Similarly, macroautophagy could be a survival adaptation isolating and degrading ROS-damaged organelles before pro-apoptotic signals are released. The damaged organelle signals for apoptosis or adaptive programs to allow survival depending on the nature and extent of damage. Pro-survival signals result in morphologically and biochemically distinct features from healthy cells that may offer a means of selecting already stressed cells and, therefore, although autophagy can trigger cell death this response is not always the best treatment outcome. Interestingly, the PS verteporfin inhibits autophagy in the dark ${ }^{[44]}$ offering the possibility of complementary combinatorial therapies in a single species.

\section{Subcellular ROS Response}

The significance of subcellular localisation of the PS and consequently ROS generation beyond the PDT effect has only recently become evident. When ROS is generated outside the cell it can lead to vascular damage including clotting and endothelial damage. This vascular mode of action has been used in phase III clinical trials for the treatment of prostate cancer. It can also be used in combination therapies where mild ROS production may alter the vascular system to promote localisation and infiltration of the tumour tissue by known chemotherapeutics.

In the extracellular matrix, ROS can be used to alter the stroma to facilitate chemotherapeutic infiltration or the cellular membrane to promote uptake. Certain types of PSs interact directly with the plasma membrane. These species are proposed to induce an effect by either modulating the embedded proteins ${ }^{[45]}$ or the phospholipid bilayer itself, ${ }^{[18,46]}$ resulting in depolarisation of the membrane and elevated cytoplasmic calcium levels ${ }^{[47]}$ and ultimately cell death by necrosis. ${ }^{[48]}$ Subcellular localisation of the PS also results in organelle membrane disruption.

When the PS is located in the lysosome, cytotoxicity is enhanced. Chlorin NPe6 and palladium WST11 are examples of agents that induce lysosomal photo-damage. Lysosomal PSs induce both promotion of autophagic stress and suppression of autophagic prosurvival functions, but ultimately cell death via apoptosis. The production of ceramide ${ }^{[49]}$ and signalling via Bid to the mitochondria initiate the process. Lysosomes have also been shown to be a potential site of localisation for aggregated and/or hydrophilic sensitisers. ${ }^{50,51]}$

When the PS is localised in the mitochondria, apoptosis is signalled via release of cytochrome c, ${ }^{[52]}$ disrupting the respiratory chain and involving caspase driven apoptosis. ${ }^{[53,54]}$ Targeting the mitochondria, as with lysosomal targeting, results in cell death, but also protective autophagy. This protection alters the efficacy of treatment such that a lysosomal sensitiser is effective at a much lower dose than a similar mitochondrial sensitiser. ${ }^{[55]}$

The endoplasmic reticulum (ER) is considered as the organelle of choice for PDT-induced cell death. The ER and the Golgi apparatus are in close proximity in the perinuclear area of the cytoplasm their functions are also linked. The ER is involved in the release of intracellular stores of calcium, ${ }^{[54]}$ but its high volume function is in the production of new biomolecules in the cell. The Golgi apparatus receives newly synthesised proteins from the ER and modifies, ${ }^{[56]}$ sorts, and packages macromolecules for cell secretion or use within the cell. It is thereby involved in metabolic control, cell communication and, inevitably, affected by oxidative stress to replace ROS damaged macromolecules. [57] However, these closely related organelles are also targets for PDT induced damage themselves ${ }^{[58-60]}$ and this phenomenon has been proposed to explain the high phototoxicity of tolyporphin ${ }^{[58]}$ and zinc(II) phthalocyanine. ${ }^{[61]}$ The ER and Golgi apparatus are suggested to be the subcellular localisation site for Foscan ${ }^{\circledR}{ }^{\left[{ }^{[62]}\right.}$

ROS production in the ER boosts protein aggregation and calcium release into the cytoplasm, signalling for apoptosis eventually via the mitochondria. In addition, direct signalling is mediated to mitochondria in contact with the ER. Singlet oxygen production in the ER leads to calureticulin and ecto-CRT release, which in turn can activate dendritic cells, stimulating the innate immune system to attack the cancer cells both localised in the treatment site and those that have migrated beyond its boundaries.

Altered function of the Golgi apparatus can signal for apoptosis. In signalling pathways involving the oligomerisation of tumour necrosis factor-related apoptosis inducing ligand receptors (TRAIL-Rs), caspase 8 can be activated signalling via the mitochondria for programmed cell death. This pro-apoptotic pathway involves signal presentation on the cell surface and therefore the Golgi as a target affects intercellular communication.

In the nucleus, PDT-mediated ROS generation leads to DNA damage. Although this damage can lead to apoptosis or in severe cases necrosis, the effect can also be mutagenic, which negates one of the clear advantages of PDT over radiotherapy and DNA-targeting chemotherapy modalities. Where PSs are known to cause DNA damage, for example methylene blue, ${ }^{[61,63]}$ clinical use involves surficial disinfection where treatment time is too short for nuclear localisation.

Whereas complete apoptosis of diseased cells was once the treatment objective in ablative PDT-treatments, heterogeneous treatments are beginning to be considered as preferential. Apoptosis is an immunologically benign method of cell death, whereas necrosis can stimulate dendritic cells to induce immunological cell death of untreated cells, including cells located distally to the treatment site. Necrosis is controlled by RIP kinases. The RIP protein forms aggregates that, when phosphorylated via caspase 8 and caspase 9 signalling, translocate to the plasma membrane to form pores and begin the necrotic process. Necrosis induces inflammation attracting neutrophils to the site (Fig. 3). Cytokine release allows the neutrophils to adhere to the endothelial walls of the vascular system such that they can invade the tumour. The outcome of this invasion depends on the tumour environment, with CD8+ cells causing tumour regression whilst other responses can lead to tumour tolerance. Finally, complete regression may be mediated by dendritic cell recognition allowing T-cell activation. As PDT elicits a dose dependant response from ROS-mediated proliferation through to necrosis, careful optimisation of the treatment parameters can either induce a complete ablative effect, ideal to treat early stage tumours, through to activation of immunological cell death for the treatment of metastasis. 


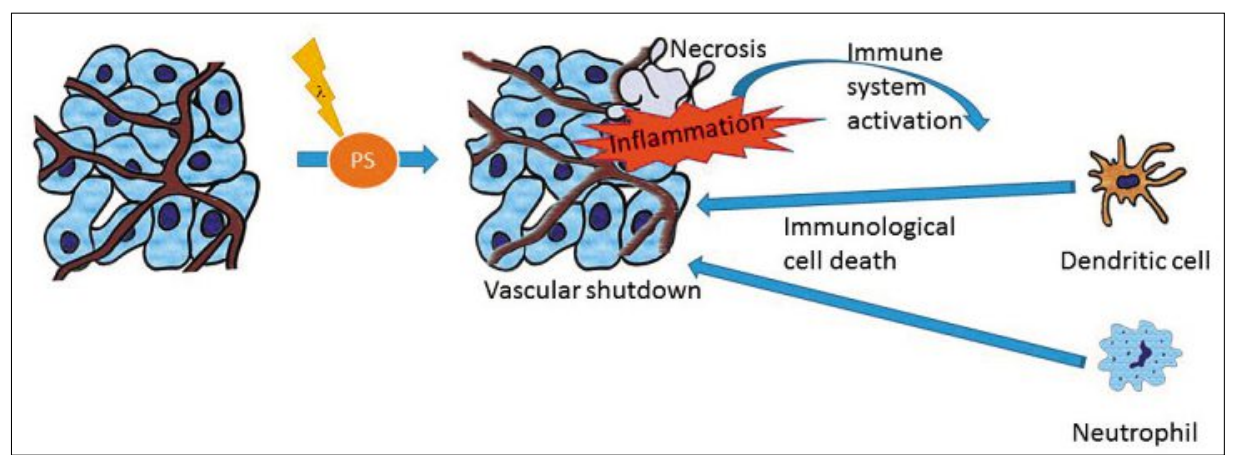

Fig. 3. Schematic representation of vascular mediated PDT. ROS in the vascular system leads to shut down and subsequent hypoxia in the treatment site. Cell death is primarily by necrosis which may lead to immune system activation, including both the maturation of dendritic cells to search for similarly diseased cells and the invasion of the treatment site by neutrophils.

\section{Novel PDT Applications}

With the ability to target photosensitive agents to a particular subcellular localisation and tailoring the photosensibility to control ROS release, the potential to expand PDT to new ablative applications and non-ablative techniques is becoming a real possibility (Fig. 2). Recent reports demonstrated how careful control of the level of ROS production could afford localised lymphatic ablation, sparing the vascular system from damage and offering a treatment modality for controlling inflammation, cancer metastasis, autoimmunity and transplant rejection. ${ }^{64]}$ Tight control of vascular damage may also be used to control bleeding, for example, in gastrointestinal mucosal vascular lesions. [65]

With increasing dose, ROS signals for proliferation, chaperone-autophagy, macroautophagy, apoptosis and then necrosis. This range of dose-dependent responses introduces an important consideration in treatment design as low level PDT could promote certain disease states, such as cancer, but on the other hand opens PDT to new applications, including regenerative, proliferative ${ }^{[66]}$ and autophagy-centred therapies, for example, in reversing incomplete autophagy associated with mitochondrial disease and aging. The proliferative effects could be applied to wound healing. ${ }^{[67]}$ Such treatments could be combined antimicrobial and disinfectant PDT applications that are currently used and under development ${ }^{[68]}$ to provide a multimodal treatment. Similarly, ablative anticancer and antimicrobial therapies may be combined into a single treatment. ${ }^{[69]}$

In eukaryotic cells, ROS levels increase during cell differentiation and activate various transcription factors identified as important for the progression and regulation of many disease states, including cancer. For example, p53, retinoblastoma gene, and phosphatase and tensin homolog are regulated by ROS. ${ }^{[25]}$ Furthermore, ROS signals via $\mathrm{NF}-\kappa \mathrm{B}$, activator protein-1, hypoxia-inducible factor- $1 \alpha$ and signal transducer and activator of transcription 3 to regulate inflammation, cell survival and growth. In the case of cancer cells, this signalling is involved in disease progression including invasion, angiogenesis and metastasis. This realisation requires critical consideration of the current ablative applications of PDT to ensure the treatment zone is sufficiently large to encompass the entirety of the diseased tissue. Certainly, decades of success in the clinic suggest this is currently the case for approved techniques.

Much less is known about ROSmediated regulation of cellular processes in prokaryotes. Data show that ROS is a powerful mode of communication between cells; both same species communication within biofilms and interspecies communication in both symbiotic and pathogenic relationships. Thus, ROS could be an important therapeutic target for microbial regulation, especially given that biofilm formation is strongly associated with pathogen resistance to chemotherapeutic agents. Low level PDT has been demonstrated to improve chemotherapeutic infiltration of eukaryotic tumours via stroma modulation and the same may be true for the biofilm capsule. Furthermore, modulation of ROS could help the host stimulate pathogen control as enteric commensal bacteria are known to affect diverse homeostatic functions of their host via ROS, including cell growth regulation, maintenance of barrier function and immunomodulation. ${ }^{[70]}$

It is speculated that the development of resistance to PS therapies should be limited by the intrinsic nature of ROS. Some cells and microbes are better equipped at processing ROS and thereby limiting ROSinduced damage, whereas certain diseased cell types are highly sensitive to these species offering another means of selectivity.

The realisation that at the treatment boundaries low levels of ROS can induce cell proliferation has caused some concerns as ablative PDT treatments must either be singularly exhaustive or part of a combination therapy, either with other treatment modalities or via stimulation of an immunological response. Combination therapies of PDT with clinically approved treatments, such as angiostatic tyrosine kinase inhibitors sunitinib, sorafenib and axitinib, ${ }^{[71]}$ are being investigated. Some strategies use PDT to induce a cytostatic effect, whereas in other cases it is used to modulate the permeability of cells to increase the potency of other drugs. ${ }^{[72]}$

\section{A New World Opened Through Accurate Light Dosimetry}

In all PDT applications, light is required to induce the therapeutic effect with the ideal PS having little or no dark toxicity. Where the drug is evenly distributed through the treatment site, light dosimetry is responsible for inducing the appropriate level of ROS for the therapeutic effect. As with the drug, the response is linked to the light dosimetry, with a greater amount of ROS and therefore cell death where there is more light through to small amounts of ROS and potentially stimulation of cell growth where the light source is at the weakest photoactive level. Any light-mediated excitation of the PS will lead to ROS production, but the therapeutic response depends on specific thresholds for the different ROS responses, which in turn depend on the cell type and status. As tumours have heterogeneous cell populations several responses will be initiated. Similarly, in other indications, different responses may be elicited in diseased versus healthy cells. Optimisation of light dosimetry can be used to achieve the most beneficial treatment outcome. For example by selecting the appropriate light wavelength, the depth of treatment can be optimised with wavelengths within the biological window maximising penetration when treating solid tumours, whereas surficial treatments, including microbial sterilisation, may benefit from using a wavelength of light that is absorbed by the naturally occurring photodynamically inactive species to limit the treatment depth. ${ }^{[7,40,66,73]}$

Although providing an even light dose over a flat and homogeneous surface is readily achievable, light dosimetry through tissues is complex, but well understood: [74] as chemical advances have explored and characterised different features of the PS, technological advances have led to a deep understanding of light dosimetry that has been translated into modelling systems able to project light propagation through tissue and a range of medical devices designed to deliver a particular dosimetry to a 
particular tissue type despite the complexity and variability. Indeed, light dosimetry is not an absolute value. In some cases, significant differences in light penetration of the same tissue can arise between different individuals. Skin tone is an obvious example. As melanin absorbs a large part of the visible spectrum, PDT using visible light in dermatological applications can have a significantly different response, in both the amount of ROS produced and the treatment depth. Internal organs also exhibit absorption variability. Studies on the bladder show $20 \%$ variation in light reflectivity between patients. ${ }^{[75]}$ Such studies reinforce the importance of in situ light dosimetry measurement and using this type of measurement allows an even dosimetry to be achieved in irregular cavities ${ }^{[76]}$ and within solid tumours. ${ }^{[77]}$ In the latter case, choice of a vascular-targeting PS means light penetration increases during treatment as blood flow reduces.

The importance of technology to ensure accurate light dosimetry can be best illustrated with an example. Initially in the development of Photofrin ${ }^{\circledR}$-PDT for high grade dysplasia of the oesophagus, a cylindrical light diffuser was positioned in the centre of the oesophageal lumen. As the oesophagus is convoluted and folded, some areas receive a higher light dose than others and, because of the threshold of the ROS response, there will be response zones with defined boundaries: in shielded folds little or no illumination leads to no response; a proliferative ROS dose may be observed in distant sites; the possibility of overdose leading to fistula where the tissue was closest to the light source; and a beneficial treatment outcome where there are appropriate PS and light doses. It was soon realised that the technique was only viable with oesophageal dilation. Inflation of the oesophagus with gas affords partial dilation, but the current state of the art is to use silicone balloon technology to dilate the oesophagus without undue pressure on the surface such that oxygenation is maintained. The balloon evenly distributes the light across its surface so there is no dosimetry variation due to distance. Such technology has been developed to allow even dosimetry in a number of natural body cavities and sites where surgery to remove diseased tissue has produced a cavity. Combined, the PS and the technology led to a successful treatment. The same is likely to be true for applying PDT to new applications; the development may not initially lie in developing new PS candidates, but in modulating light dosimetry to move the treatment outcome from ablative to proliferative.

\section{Summary}

The molecular mechanism of PDT involving ROS is now well established. Drug design has led to a series of approved PSs that have different modes of action. Currently, the focus is on tissue ablation, with ROS production maximised over the treatment area. Unsurprisingly, there have been some clear success stories heralding the notion PDT has come of age, most notably the wide use of ablative PDT in ophthalmology and dermatology. On the other hand, there are a number of approved treatments, particularly those for cholangiocarcinoma and high grade dysplasia in Barrett's oesophagus, that are rarely used in the clinic. Whilst there are many reasons behind the under-representation of certain ablative clinical applications, the comparative lack of research into other ROS-modulating treatments is harder to explain and is certainly a missed opportunity. Applications of the currently approved drugs to challenging health issues may not require further drug design; rather it may be achievable by modulating the light dosimetry alone. Such applications may include promoting cell regeneration and proliferation in wound healing; stimulating the dendritic immune response against diffusely located diseased cells; or promotion of autophagy to treat mitochondrial disease. These therapies could be achievable based on existing PS with optimised control of light dosimetry, resulting in rapid expansion of PDT into new clinical domains. And whilst these applications remain unexplored, PDT will not reach its full clinical potential.

Received: November 28, 2014

[1] I. Ashur, R. Goldschmidt, I. Pinkas, Y. Salomon, G. Szewczyk, T. Sarna, A. P. Scherz, J. Phys. Chem. A 2009, 113, 8027.

[2] A. L. Edinger, C. B. Thompson, Curr. Opin Cell Biol. 2004, 16, 663 .

[3] M. Price, S. R. Terlecky, D. Kessel, Photochem. Photobiol. 2009, 85, 1491.

[4] R. Bonnett, G. Martinez, Tetrahedron 2001, 57, 9513

[5] R. Bonnett, Chem. Soc. Rev. 1995, 24, 19

[6] E. S. Nyman, P. H. Hynninen, J. Photochem. Photobiol. B 2004, 73, 1

[7] K. Lang, J. Mosinger, D. M. Wagnerová, Coordin. Chem. Rev. 2004, 248, 321.

[8] R. Bonnett, P. Charlesworth, B. D. Djelal, S. Foley, D. J. McGarvey, T. G. Truscott, J. Chem. Soc. Perkin Trans. 2 1999, 2, 325.

[9] C. E. Wayne, R. P. Wayne, 'Photochemistry', Vol. 39. Oxford, UK, Oxford Science, Oxford University Press, 1996; 'Photophysics', Oxford Chemistry Primers, chapter 3, p. 41

[10] A. Gilbert, J. Baggott, 'Essentials of Molecular Photochemistry' Blackwell Scientific, Oxford, UK, 1991, p. 8-9.

[11] G. R. Mufti, P. J. Shah, M. C. Parkinson, P. R. Riddle, Br. J. Urol. 1990, 65, 173; W. B. Gill, J. L. Huffman, E. S. Lyon, D. H. Bagley, H. W. Schoenberg, F. H. Straus, 2nd, Cancer 1984, 53, 2724.
[12] F. Wilkinson, W. P. Helman, A. B. Ross, J. Phys. Chem. Ref. Data 1993, 22, 113.

[13] D. M. Guldi, T. D. Mody, N. N. Gerasimchuk, D. Magda, J. L. Sessler, J. Am. Chem. Soc. 2000, 122,8289 .

[14] T. J. Dougherty, C. J. Gomer, B. W. Henderson, G. Jori, D. Kessel, M. Korbelik, J. Moan, Q. Peng, J. Natl. Cancer Inst. 1998, 90, 889.

[15] M. G. Alvarez, N. B. R. Vittar, F. Principe, J. Bergesse, M. C. Romanini, S. Romanini, M. Bertuzzi, E. N. Durantini, V. Rivarola, Photodiag. Photodyn. Ther. 2004, 1, 335; L. Collins-Gold, N. Feichtinger, T. Wärnheim, Modern Drug Discov. 2000, 3, 44 .

[16] M. García-Díaz, M. Kawakubo, P. Mroz, M. L. Sagristà, M. Mora, S. Nonell, M. R. Hamblin, J. Controlled Release 2012, 162, 355.

[17] E. G. Randles, P. R. Bergethon, Langmuir 2013, $29,1490$.

[18] W. Guo, C. Yang, L. Cui, H. Lin, F. Qu, Langmuir 2014, 30, 243.

[19] C. Banerjee, S. Maiti, M. Mustafi, J. Kuchlyan, D. Banik, N. Kundu, D. Dhara, N. Sarkar, Langmuir 2014, 30, 10834.

[20] J. Shi, Z. Xiao, N. Kamaly, O. C. Farokhzad, Acc. Chem. Res. 2011, 44, 1123.

[21] Y. Wang, Y. Liu, G. Li, J. Hao, Langmuir 2014, 30,6419

[22] S. V. Lepeshkevich, M. V. Parkhats, A. S. Stasheuski, V. V. Britikov, E. S. Jarnikova, S. A. Usanov, B. M. Dzhagarov, J. Phys. Chem. A 2014, 118, 1864

[23] J. Comas-Barceló, B. Rodríguez-Amigo, S. Abbruzzetti, P. del Rey-Puech, M. Agut, S. Nonell, C. Viappiani, RSC Adv. 2013, 3, 17874.

[24] R. Hudson, M. Carcenac, K. Smith, L. Madden, O. J. Clarke, A. Pelegrin, J. Greenman, R W. Boyle, B. J. Cancer 2005, 92, 1442; N. Malatesti, K. Smith, H. Savoie, J. Greenman, R. W. Boyle, Int. J. Oncol. 2006, 28, 1561; C. Staneloudi, K. A. Smith, R. Hudson, N. Malatesti, H. Savoie, R. W. Boyle, J. Greenman, Immunol. 2007, 120, 512

[25] E. Rosàs, P. Santomá, M. Duran-Frigola, B. Hernandez, M.C. Llinàs, R. Ruiz-González, S. Nonell, D. Sánchez-García, E. R. Edelman, M. Balcells, Langmuir 2013, 29, 9734.

[26] S. Zheng, P. L. M. Lynch, T. E. Rice, T. S. Moody, H. Q. N. Gunaratne, A. Prasanna de Silva, Photochem. Photobiol. Sci. 2012, 11, 1675.

[27] C. Herrero, L. Batchelor, A. Baron, S. El Ghachtouli, S. Sheth, R. Guillot, B. Vauzeilles, M. Sircoglou, T. Mallah, W. Leibl, A. Aukauloo, Eur. J. Inorg. Chem. 2013, 4, 494.

[28] K. Ouhenia-Ouadahi, R. Métivier, S. Maisonneuve, A. Jacquart, J. Xie, A. Léaustic, P. Yu, K. Nakatani, Photochem. Photobiol. Sci. 2012, 11, 1705.

[29] R. Ruiz-González, R. Zanocco, Y. Gidi, A. L. Zanocco, S. Nonell, E. Lemp, Photochem. Photobiol. 2013, 89, 1427.

[30] T. Lammers, S. Aime, W. E. Hennink, G. Storm, F. Kiessling, Acc. Chem. Res. 2011, 44, 1029.

[31] L. B. Josefsen, R. W. Boyle, Theranostics 2012, 2,916 .

[32] H. Ali, J. E. van Lier, Chem. Rev. 1999, 99, 2379.

[33] D. J. Ball, S. R. Wood, D. I. Vernon, J. Griffiths, T. M. A. R Dubbelman, S. B. Brown, $J$. Photochem. Photobiol. B 1998, 45, 28.

[34] V. Mantareva, V. Kussovski, I. Angelov, E. Borisova, L. Avramov, G. Schnurpfeil, D. Wöhrle, Bioorg. Med. Chem. 2007, 15, 4829.

[35] L. Ding, J. Balzarini, D, Schols, B. Meunier, F. B. De Clercq, Biochem. Pharmacol. 1992, $44,1675$.

[36] S. H. Selman, J. A. Hampton, A. R. Morgan, R. W. Keck, A. D. Balkany D. Skalkos, Photochem. Photobiol. 1993, 57, 681.

[37] M. Oschner, J. Photochem. Photobiol. B 1996, 32, 3; K. Schieweck, H.-G. Capraro, U. Isele, 
P. van Hoogevest, M. Ochsner, T. Maurer, E. Batt, Proc. SPIE 1994, 107, 2078

[38] A. S. Sobolev, E. F. Stranadko, Int. Photodyn. Ther. 1997, 1, 2; V. V. Sokolov, V. I. Chissov, E. V. Filonenko, R. I. Yakubovskaya, G. M. Sukhin, M. G. Galpern, G. N. Vorozhtsov, A. V. Gulin, M. B. Zhitkova, N. N. Zharkova, D. N. Kozlov, V. V. Smirnov, Proc. SPIE 1995 , 23325, 364; N. N. Zharkova, D. N. Kozlov, V. V. Smirnov, V. V. Sokolov, V. I. Chissov, E. V. Filonenko, G. M. Sukhin, M. G. Galpern, G. N. Vorozhtsov, Proc. SPIE 1995, 2325, 400.

[39] W. M. Sharman, C. M. Allen, J. E. van Lier, Drug Discovery Today 1999, 4, 507; I. J. MacDonald, T. J. Dougherty, J. Porphyr. Phthalocy. 2001, 5, 105.

[40] 'Efficacy, Safety and Quality of Life After TOOKAD $^{\circledR} \quad$ Soluble Vascular Targeted Photodynamic Therapy for Localized Prostate Cancer (PCM304)', Steba Biotech S.A., ClinicalTrials.gov Identifier: NCT01875393.

[41] K. Svanberg, I. Wang, S. Colleen, I. Idvall, C. Ingvar, R. Rydell, D. Jocham, H. Diddens, S. Bown, G. Gregory, Acta Radiologica 1998, $39,2$.

[42] D. Gozuacik. A. Kimchi, Oncogene 2004, 23, 2891; L. Yu, A. Alva, H. Su, P. Dutt, E. Freundt, S. Welsh, E. H. Baehrecke, M. J. Lenardo, Science 2004, 304, 1500; E. L. Eskelinen, Cell Death Differ. 2005, 12, 1468; Y. Tsujimoto S. Shimizu, Cell Death Differ. 2005, 12, 1528; R. A. Lockshin, Z. Zakeri, Int. J. Biochem. Cell Biol. 2004, 36, 2405; A. Guillon-Munos, M. X. van Bemmelen, P. G. Clarke, Apoptosis 2005, 10, 1031 .

[43] D. Kessel, J. Natl. Compr. Canc. Netw. 2012, 10, S-56.

[44] E. Donohue, A. Tovey, A. W. Vogl, S. Arns, E. Sternberg, R. N. Young, M. Roberge, J. Biol. Chem. 2011, 286, 7290.

[45] N. Wang, Y. Liu, M. Xia Xie, Z. Jie Cui, J. Photochem. Photobiol. B 2003, 71, 27.

[46] J. Kim, O. Amorim Santos, J.-H. Park, J. Controlled Release 2014, 191, 98.

[47] K. G. Specht, M. A. J. Rodgers, Biochim. Biophys Acta (BBA) 1991, 1070, 60.

[48] W. S. Ahn, S. M. Bae, S. W. Huh, J. M. Lee, S. E. Namkoong, S.-J. Han, C. K. Kim, J.-K. Kim,
Y.-W. Kim, Int. J. Gynecological Cancer 2004, 14,475

[49] H. Sawai, Y. A. Hannun, Chem. Phys. Lipids 1999, 102, 141 .

[50] M. Gèze, P. Morlière, J. C. Mazière, K. M. Smith, R. Santus, J. Photochem. Photobiol. B 1993, 20, 23.

[51] K. Berg, J. Moan, Photochem. Photobiol. 1997, $65,403$.

[52] L. Y. Xue, S. M. Chiu, N. L. Oleinick, Exp. Cell Res. 2001, 263, 145.

[53] C. M. Yow, J. Y. Chen, N. K. Mak, N. H. Cheung, A. W. Leung, Cancer Lett. 2000, 157123.

[54] M. Terasaki, J. Song, J. R. Wong, M. J. Weiss, L. B. Chen, Cell 1984, 38, 101.

[55] M. E. Rodriguez, P. Zhang, K. Azizuddin, G. B. Delos Santos, S. M. Chiu, L. Y. Xue, J. C. Berlin, X. Peng, H. Wu, M. Lam, A. L. Nieminen, M. E. Kenney, N. L. Oleinick, Photochem. Photobiol. 2009, 85, 1189 .

[56] B. Short, F. A. Barr, Curr Biol. 2000, 10, R583.

[57] Z. Jiang, Z. Hu, L. Zeng, W. Lu, H. Zhang, T. Li, H. Xiao, Free Rad. Biol. Med. 2001, 50, 907.

[58] J. Y. Matroule, G. Bonizzi, P. Morlière, N. Paillous, R. Santus, V. Bours, J. Piette, J. Biol. Chem. 1999, 274, 2988.

[59] P. Morlière, J. C. Mazière, R. Santus, C. D. Smith, M. R. Prinsep, C. C. Stobbe, M. C. Fenning, J. L. Golberg, J. D. Chapman, Cancer Res. 1998, 58, 3571.

[60] C. Fabris, G. Valduga, G: Miotto, L. Borsetto, G. Jori, S. Garbisa, E. Reddi, Cancer Res. 2001, 61, 7495.

[61] A. P. Castano, T. N. Demidova, M. R. Hamblin, Photodiag. Photodyn. Ther. 2004, 1, 279.

[62] M.-H. Teiten, L. Bezdetnaya, P. Morlière, R. Santus, F. Guillemin, Br. J. Cancer 2003, 88, 146.

[63] J. L. Sessler, N. A. Tvermoes, J. Davis, P. Anzenbacher, Jr., K. Jursíková, W. Sato, D. Seidel, V. Lynch, C. B. Black, A. Try, B. Andrioletti, G. Hemmi, T. D. Mody, D. J. Magda, V. Král, Pure Appl. Chem. 1999, 71, 2009.

[64] W. W. Kilarski, A. Muchowicz, M. Wachowska, R. Mężyk-Kopeć, J. Golab, M. A. Swartz, P. Nowak-Sliwinska, Angiogenesis 2014, 17, 347. [65] H. Qiu, Y. Mao, Y. Gu, Y. Wang, J. Zhu, H.
Zeng, Photodiag. Photodynam. Ther. 2012, 9, 109.

[66] S.C. Gupta, D. Hevia, S. Patchva, B. Park, W. Koh, B. B. Aggarwal, Antioxid. Redox Signal. 2012, 16, 1295.

[67] R. Spitler, M. W. Berns, J. Biomed. Opt. 2014, 19, 038001.

[68] C. Vassena, S. Fenu, F. Giuliani, L. Fantetti, G. Roncucci, G. Simonutti, C. L. Romanò, R. De Francesco, L. Drago, Int. J. Antimicrob. Agents 2014, 44, 47.

[69] H. Qiu, Y. Mao, Y. Gu, J. Zhu, Y. Wang, J. Zeng, N. Huang, Q. Liu, Y. Yang, J. Photochem. Photobiol. B 2014, 130, 305.

[70] P. A. Swanson II, A. Kumara, S. Samarin, M. Vijay-Kumar, K. Kundu, N. Murthy, J. Hansen, A. Nusrat, A. S. Neish, PNAS 2011, 108, 8803.

[71] A. Weiss, J. R. van Beijnum, D. Bonvin, P. Jichlinski, P. J. Dyson, A. W. Griffioen, P. Nowak-Sliwinska, J. Cell. Mol. Med. 2014, $18,480$.

[72] L. M. Giroldo, M. P. Felipe, M. A. de Oliveira, E. Munin, L. P. Alves, M. S. Costa, Lasers Med. Sci. 2009, 24, 109; Y. Wang, M. Gonzalez, C. Cheng, A. Haouala, T. Krueger, S. Peters, L. A. Decosterd, H. van den Bergh, J. Y. Perentes, H. B. Ris, I. Letovanec, E. Debefve, Lasers Surg. Med. 2012, 44, 318 .

[73] M. Oschner, J. Photochem. Photobiol. B 1997, 39,1 .

[74] S. T. Flock, M. S. Patterson, B. C. Wilson, D. R. Wyman, Lasers Med. Sci. 1986, 1, 235; W. M. Star, Phys. Med. Biol. 1997, 42, 763; A. E. Karsten, A. Singh, M. W. Braun, Lasers Med. Sci. 2012, 27, 79; J. Guo, Z. Li, W. Xie, H. Chen, G.-X. Weng, H. Li, Proc. SPIE 2014, 9230.

[75] J. P. A. Marijnissen, W. M. Star, H. J. A in 't Zandt, M. A. D'Hallewin, L Baert, Phys. Med. Biol. 1993, 38, 567

[76] H.-B. Ris, Lung Cancer 2005, 49, S65.

[77] C. M. Moore, A. R. Azzouzi, E. Barret, A. Villers, G. Muir, N. Barber, J. Trachtenberg, N. Arumainayagam, B. Gaillac, C. Allen, A. Schertz, M. Emberton, BJU Int. 2014, 1464. 